Delroisse et al. 2020

\title{
Integument transcriptome profile of the European sea cucumber Holothuria forskali (Holothuroidea, Echinodermata)
}

Jérôme Delroisse*, Marie Bonneel, Mélanie Demeuldre, Igor Eeckhaut, Patrick Flammang*

University of Mons - UMONS, Research Institute for Biosciences, Biology of Marine Organisms and Biomimetics Unit, 23 Place du Parc, 7000 Mons - Belgium

Jerome.Delroisse@umons.ac.be

Marie.Bonneel@umons.ac.be

Melanie.Demeuldre@gmail.com

Igor.Eeckhaut@umons.ac.be

Patrick.Flammang@umons.ac.be

* Correspondence: Jerome.Delroisse@ umons.ac.be, Patrick.Flammang@umons.ac.be

\section{Abstract}

In non-model organisms, Next Generation Sequencing (NGS) technology improve our ability to analyze gene expression and identify new genes or transcripts of interest. In this research, paired-end Illumina HiSeq sequencing has been used to describe a composite transcriptome based on two libraries generated from dorsal and ventral integuments of the European sea cucumber Holothuria forskali (Holothuroidea, Echinodermata). A total of 43,044,977 million HQ reads were initially generated. After de novo assembly, a total of 111,194 unigenes were predicted. On all predicted unigenes, 32,569 show significant matches with genes/proteins present in the reference databases. Around 50\% of annotated unigenes were significantly similar to sequences from the purple sea urchin Strongylocentrotus purpuratus genome. Annotation analyses were performed on predicted unigenes using public reference databases. These RNA-seq data provide an interesting resource for researchers with a broad interest in sea cucumber biology.

Keywords: sea cucumber, echinoderm, RNA-seq, transcriptome, integument 
Delroisse et al. 2020

37

38

39

40

41

42

43

44

45

46

47

\section{Introduction}

Holothuroidea, also known as sea cucumbers, are worm-like soft-bodied marine organisms belonging to the echinoderm phylum and present worldwide. The class counts more than 1,600 species and several species are highly marketable as a food product in East Asian countries [1]. Nowadays, to continuously supply the high demand from the markets, new non-target species from the northern hemisphere are being fished and traded [2]. Sea cucumbers also possess a wide range of bioactive compounds that can potentially be used in the pharmaceutical industry [3, 4]. These compounds may present interesting biological activities (e.g. antioxidant, anticoagulant and wound healing, anti-inflammatory, antitumor or antimicrobial [57]). The sea cucumber Holothuria forskali is a common species found in the Eastern Atlantic Ocean and the Mediterranean Sea. This detritivore holothuroid of the family of Holothuriidae is found at shallow depth and is considered as a keystone species in its environment [8].

For non-model, or emerging model, marine organisms, Next Generation Sequencing technologies offer an opportunity for rapid access to sequence data and genetic information. Multiple echinoderm transcriptomes emerged in the literature in the last years $[9,10]$, bringing important molecular information on highly diverse biological processes such as development [11], regeneration [12], sensory perception [13], adhesion [14] or evolution [15]. In the present study, paired-end Illumina HiSeq sequencing technology has been used to generate an integument transcriptome of the sea cucumber $H$. forskali. More specifically, dorsal (i.e. bivium) and ventral (i.e. trivium) integuments were investigated separately as they were considered as functionally distinct. The transcriptome of the integument will be a valuable resource to better understand biological mechanisms occurring in this specific tissue and should positively impact future studies focusing on the species $H$. forskali but also on sea cucumbers in general. In particular, the distinction between ventral and dorsal integument libraries allows an emphasis on biological processes specific to both sides of the integument (e.g. the ventral integument includes tube feet involved in adhesion to the substratum, the dorsal integument is thought to be involved in sensory perception).

\section{Data description}

\subsection{Animal collection and RNA isolation}


Delroisse et al. 2020

72 Adult individuals of $H$. forskali were collected in the vicinity of the Marine Station of

73 Banuyls-sur-Mer (France) in summer 2014. After dissection, the dorsal and ventral

74 body wall were separated and the integument (i.e. dermis and epidermis) was cut into

75 small pieces. The tissues were treated with fresh Trizol@ solution and RNA

76 extractions were performed according to the Trizol® manufacturer's protocols. The

77 quality of RNA extracts was checked using 1.2 M TAE agarose gel electrophoresis

78 and spectrophotometric measurements using a Nanodrop spectrophotometer (LabTech

79 International). RNA quality was finally assessed by size chromatography using an

80 Agilent 2100 Bioanalyzer.

82 Table 1. MIxS descriptors of the study

\begin{tabular}{ll}
\hline Item & Description \\
\hline Classification & Eukaryota; Animalia; Echinodermata; Echinozoa; \\
& Holothuroidea; Actinopoda; Holothuriida; Holothuriidae; \\
& $\begin{array}{l}\text { Holothuria; Holothuria (Panningothuria); Holothuria } \\
\text { (Panningothuria) forskali }\end{array}$ \\
Submitted_to_insdc & Yes (SRA, TSA) \\
Investigation_type & Eukaryote transcriptome \\
Project_name & Integument transcriptome of the sea cucumber Holothuria \\
& forskali \\
Lat_lon & $42^{\circ} 29^{\prime} 01^{\prime \prime}$ N, 3007'44" E \\
Geo_loc_name & Banuyls-sur-Mer, France (Observatoire Océanologique) \\
Collection_date & 2014 \\
Env_Biome & Seawater (ENVO:00002149) \\
Env_Feature & Rocky shore (ENVO:01000428) \\
Env_Material & Seawater (ENVO:00002149) \\
Env_Package & Water \\
Temp & NA \\
Salinity & NA \\
Collected_by & Staff members of the “Observatoire Océanologique” of \\
& Banuyls-sur-Mer \\
Sequencing method & Illumina HiSeq \\
Assembly method & Trinity software \\
Organ or tissue & Adult: dorsal integument, ventral integument \\
source & \\
Database name & NCBI \\
Project name & PRJNA481065 \\
Sample names & SAMN09655902, SAMN09655903 \\
\hline
\end{tabular}

\subsection{Library preparation and sequencing}

85

86 Total RNA Samples were sent to a commercial sequencing service provider (Beijing

87 Genomics Institute, Hong Kong). RNA samples were treated with DNase I and poly- 


\section{Delroisse et al. 2020}

88 (A) mRNAs were then enriched using oligo(dT) magnetic beads and fragmented into 89 short pieces (around $200 \mathrm{bp}$ ). Random hexamer-primers were used to synthesize the 90 first-strand cDNA using the short fragments as templates. DNA polymerase I was 91 used to synthesize the second-strand cDNA. After purification, Double-stranded 92 cDNAs were subjected to end reparation and 3' single adenylation. Sequencing 93 adaptors were ligated to the adenylated fragments, which were then enriched by PCR 94 amplification. High-throughput sequencing was conducted using the Illumina 95 HiSeq $^{\mathrm{TM}} 2000$ sequencing platform to generate $100 \mathrm{bp}$ paired-end reads. Sequencing 96 was performed according to the manufacturer's instructions (Illumina, San Diego, 97 CA).

\subsection{Data processing and de novo assembly}

100

The quality of the new transcriptomes was checked using the software FastQC

102 (www.bioinformatics.babraham.ac.uk). An initial data cleaning was required to obtain 103 clean reads that were used for the analyses. The cleaning step was performed by the 104 sequencing service provider. It includes (i) the adaptor removal as well as (ii) the 105 application of a filtering criterion to remove reads with more than $5 \%$ of unknown 106 bases and low-quality reads (reads that comprise more than $20 \%$ low-quality bases, 107 i.e. base quality $\leq 10$ ). The Q20 percentages (i.e. base quality more than 20) were superior to $96,95 \%$ for both datasets.

109 After the initial cleaning step, the remaining 21,884,354 (ventral integument 110 library) and 21,160,623 (dorsal integument library) clean reads were used to assemble

111 the $H$. forskali integument transcriptome using the Trinity software (release 112 20130225) [16]. The following parameters were used: seqType fq, min_contig_length 113 100, min_glue 3, group_pairs_distance 160, path_reinforcement_distance 95, 114 min_kmer_cov 3.

115 For the ventral transcriptome, 216,144 contigs were generated with an average 116 of 377 base pairs (bp) while 187,773 contigs were generated for the dorsal 117 transcriptome with an average length of $391 \mathrm{bp}$. The N50 (i.e. median contig size) 118 was of $780 \mathrm{bp}$ for the ventral contig set and $814 \mathrm{bp}$ for the dorsal contig set.

119 Using paired end information and gap filling, contigs were further assembled 120 into 111,194 unique sequences (i.e. non-redundant sequences or unigenes) with a 
Delroisse et al. 2020

121 mean length of 1,048 bp including 40,067 clusters and 71,127 singletons. Numerical

122 data are summarized in Supplementary Table S1.

123 Unigenes were separated into clusters (similarity among overlapping

124 sequences is superior to $94 \%$ ) and singletons (unique unigenes). The clustering was

125 performed using the TIGR Gene Indices Clustering (TGICL) tools (v2.1, parameters:

$126-l 40-c 10-v$ 20) [17] followed by Phrap assembler (www.phrap.org, release 23.0,

127 parameters : repeat stringency 0.95, minmatch 35, minscore 35). Various quality

128 assembly criteria were evaluated such as (i) the contig/unigene size distribution and

129 the (ii) of the read distribution when realigned to unigenes using SOAP aligner

130 (Release 2.21, parameters: - $m 0$-x 500 -s 40 -l 35 -v 5 -r 1) [18]. Length distribution

131 of contigs and unigenes are presented in Supplementary Figure S1.A-D. In addition,

132 more than $82 \%$ of transcriptome unigenes from the integument of $H$. forskali were

133 realigned by more than 5 reads (Supplementary Figure S1.E).

\subsection{Unigene annotation, classification and comparative gene expression}

137 Transcriptome completeness was evaluated using BUSCO (v3.0.2) analyses on 138 assembled unigenes (Supplementary Figure S2) [19]. Scores were calculated using 139 Eukaryota_odb9 lineage data. BUSCO analyses showed that $91.1 \%$ complete BUSCO 140 groups were detected in the final gene set (84.2\% in the dorsal transcriptome, $77.3 \%$ 141 in the ventral unigenes). In detail, out of the 303 evaluated BUSCOs from the 142 Eukaryota dataset, only $8.3 \%$ were fragmented and $0.6 \%$ were missing.

143 Unigenes were compared to online databases NCBI non-redundant protein 144 and nucleotide databases (NR/NT, www.ncbi.nlm.nih.gov, release 20130408), Swiss145 Prot database (www.expasy.ch/sprot, release 2013_03), Kyoto Encyclopedia of Genes 146 and Genome (KEGG, release 63.0) (www.genome.jp/kegg) and Cluster of 147 Orthologous Groups (COG) of proteins (www.ncbi.nlm.nih.gov/COG, release148 20090331) using BLASTx with a E-value threshold of $10^{-5}$, and in the nucleic-acid 149 database (NCBI NT) by BLASTn with the same cutoff. When different databases 150 returned inconsistent results, they were prioritized in the following order: NR, 151 SwissProt, KEGG, COG. When a unigene did not align with any of the reference 152 sequences, ESTScan was used to predict candidate coding regions and determine the 153 direction of the coding sequence in the unigene [20].

154 On the 111,194 unigenes, 32,569 show significant matches with reference 
Delroisse et al. 2020

155 databases: 30,625 to NR, 10,397 to NT, 25,400 to Swiss-Prot, 22,813 to KEGG,

15611,125 to COG and to 13,833 GO (Figure 1.A, Supplementary Table S2 for the

157 complete unigene annotation table). The E-value distribution is presented in Figure

158 1.B. This E-value distribution of the "top matches" in the NR (NCBI) database

159 showed that more than $57 \%$ of the mapped unigenes have strong homology (E-value

$\left.160<1.0 \mathrm{e}^{-30}\right)$, whereas $44 \%$ of the homologous sequences presented E-values ranging

161 from $1.0 \mathrm{e}^{-05}$ to $1.0 \mathrm{e}^{-30}$ (Figure 1.B). The sequence similarity distribution indicates that

$16221 \%$ of the sequences have a similarity higher than $60 \%$ (Figure 1.C). Many unigenes

163 were similar the genes found in the sea urchin S. purpuratus (Figure 1.D) as observed

164 in other echinoderm-focused transcriptomic studies [11, 13].

165

166

167

168

169

170

171

172

173

174

175

176

177

178

179

180

181

182

183

184

185

186

187

188

189

190

191

Figure 1. Annotation statistics of the integument transcriptome from Holothuria forskali. (A) Summary of the functional annotation of the unigenes for NR, NT, Swiss-Prot, KEGG, COG, GO databases. (B) "E-value distribution" of the top BLAST hits for unigenes (E-values $<\mathrm{e}^{-5}$ ). (C) "Similarity distribution" of BLAST hits of each unigene compared to the NR (NCBI) database. (D) "Species distribution" of the top BLAST hits for all unigenes.

To investigate the enzymatic diversity of both ventral and dorsal transcriptomes, additional annotation analyses were performed using the PRIAM database [21] implemented in the webtool FunctionAnnotator [22] using a stricter Evalue threshold (cutoff of $10^{-10}$ ) (Supplementary Table S3).

On a total of 111,194 predicted unigenes, 30,104 were only found in the ventral integument transcriptome and 27,884 only in the dorsal integument transcriptome while 53,206 were detected in both transcriptomes. A comparative gene expression analysis was performed by mapping FPKM values (i.e. $\log _{10}$ [FPKM value ventral integument transcriptome]) against $\log _{10}$ [FPKM value dorsal integument transcriptome]), calculated for all predicted unigenes (Figure 2.A). However, it has to be stated that the transcriptomes have been generated in the purpose of new gene discovery and no biological or technical replication was performed as a part of the study. Based on a specific threshold ( $\mid \log _{2}[$ Fold change] $\mid \geq 1), 20,488$ unigenes were found to be upregulated in the ventral integument transcriptome against 14,087 in the dorsal integument transcriptome (Figure 2.B).

Figure 2. Comparative gene expression in $H$. forskali ventral and dorsal integuments. A. FPKM distribution of both transcriptomes. Upregulated and downregulated are color coded. Selection was based on the threshold: $\mid \log _{2}[$ Ratio] $\mid>=1$ (i.e. 
Delroisse et al. 2020

192

193

194

195

196

197

198

199

200

201

202

203

204

205

206

207

208

209

210

211

212

213

214

215

216

217

218

219

220

221

222

223

224

$\log _{2}[F P K M$ ventral +0.1$) /(F P K M$ dorsal +0.1$\left.)\right]$ B. Comparison of the differentially expressed unigenes between the ventral and dorsal integument transcriptomes.

The 10 most expressed unigenes of both transcriptomes correspond to various subunits of the "cytochrome c oxidase", a "thymosin beta-4", an "alpha-1 collagen precursor", a "ferritin" and several non-annotated unigenes (Supplementary Table S4). An "epidermal growth factor" is also specifically present within the 10 most expressed unigenes of the ventral transcriptome.

The 10 most differentially expressed unigenes (i.e. maximum or minimum $\log _{2}$ [FPKM fold change]) in both transcriptomes are listed in the Supplementary Table S5. Most of the "10 most differentially expressed unigenes" with the highest expression in the dorsal transcriptome are not annotated. The 10 most differentially expressed unigenes with the highest expression in the ventral transcriptome correspond to a "rtoA-like", an "hyalin-like", a "farnesoic acid o-methyltransferaselike", a "lactadherin-like", a "rhamnose-binding lectin-like" and several nonannotated unigenes. Several of these actors are likely to be specifically expressed in tube feet and involved in tube foot adhesion such as the "hyalin-like" and the "farnesoic acid o-methyltransferases-like". Hyalin proteins are fibrillar glycoproteins involved in cell adhesion and expressed, for example, in the sea-urchin S. purpuratus embryo [23]. Various "farnesoic acid o-methyltransferases-like" have recently been identified in footprint produced by sea star tube feet indicating a probable implication in the adhesive material elaboration [24].

For descriptive purposes, and based on PRIAM annotation, the 10 most differentially expressed predicted enzyme-coding transcripts are listed in the Supplementary Table S6. The most differentially expressed predicted enzyme-coding transcripts within the ventral integument transcriptome are several "Protein-tyrosinephosphatases", a "Guanylate cyclase", a "NAD+ ADP-ribosyltransferase", a "Proteinserine/threonine kinase", a "CDP-diacylglycerol--inositol 3-phosphatidyltransferase" and a "Creatine kinase". Within the dorsal integument transcriptome, the most differentially expressed enzyme-coding transcripts are a "Lysozyme", an "Exo-alphasialidase", a "superoxide dismutase", "DNA-directed RNA polymerase", "Peptidylprolyl isomerase", "Protein disulfide-isomerase", "RNA helicase", "Nicotinamide-nucleotide adenylyltransferase" and an "Amidase". 
Delroisse et al. 2020

\section{2.5. Data accessibility}

227 The Illumina derived short-read files are available at the NCBI Sequence Read

228 Archive under the study accession number SAMN09655902 and SAMN09655903

229 (Bioproject $\mathrm{N}^{\circ}$ PRJNA481065). This Transcriptome Shotgun Assembly project has

230 been deposited at DDBJ/EMBL/GenBank under the accession GIPR00000000. The

231 version described in this paper is the first version, GIPR01000000.

\section{Acknowledgments}

234 J.D. and P.F. are respectively Postdoctoral Researcher and Research Director of the

235 Fund for Scientific Research of Belgium (F.R.S.-FNRS). M.B. and M.D. are FRIA

236 PhD students (F.R.S.-FNRS). The work was supported in part by $(i)$ a PDR-WISD

237 project $\left(\mathrm{n}^{\circ} 29101409\right)$ from the F.R.S.-FNRS as well as the (ii) the FP7 European

238 project BYEFOULING (Grant Agreement $n^{\circ}$ 612717). This study is a contribution

239 from the "Centre Interuniversitaire de Biologie Marine" (CIBIM).

\section{Competing interests}

The authors declare that they have no competing interests.

Authors' contributions

245 J.D. performed the experiments and data analyses. J.D., M.B., M.D., P.F. conceived

246 and designed the experiments. J.D. wrote the first draft of the manuscript. All authors

247 revised the manuscript. 
Supplementary Figure S1. Distribution of contigs (A-B) and unigenes (C-D) in ventral and dorsal Holothuria forskali integument transcriptomes, respectively. The length of contigs and unigenes ranged from $200 \mathrm{bp}$ to more than 3,000 bp. (E) Assessment of assembly quality using the distribution of unique mapped reads on the assembled unigenes.

Supplementary Figure S2. Transcriptome completeness evaluation on assembled unigenes using BUSCO.

Supplementary Table S1. Data description of the Holothuria forskali integument transcriptomes. A. Description of the sequencing output. Q20 percentage is the proportion of nucleotides with quality value larger than 20 in reads. GC percentage is the proportion of guanidine and cytosine nucleotides among total nucleotides. B. Summary statistics of transcriptome assembly.

263 Supplementary Table S2. Unigene annotation using NT, NR, GO, COG and 264 KEGG databases (E-value threshold: $10^{-5}$ ).

Supplementary Table S3. Enzyme unigene annotation using PRIAM database (Evalue threshold: $10^{-10}$.

267 Supplementary Table S4. The 10 most expressed unigenes in the ventral (A) and 268 dorsal (B) integument transcriptomes of Holothuria forskali with their corresponding annotation.

Supplementary Table S5. The 10 most differentially expressed unigenes (i.e. maximum or minimum $\log _{2}$ [FPKM fold change]) in the ventral (A) and dorsal (B) integument transcriptome of Holothuria forskali with their corresponding annotation.

Supplementary Table S6. The 10 most differentially expressed predicted enzymecoding transcripts in the ventral (A) and dorsal (B) integument transcriptomes of Holothuria forskali.

Funding information This work was supported by the F.R.S.-FNRS (PDR-WISD project, grant number: 29101409) and by an FP7 European project (BYEFOULING, grant number: 612717).

\section{Compliance with ethical standards}

Conflict of interest The authors declare that they have no conflict of interest.

Ethical approval All animal collection and utility protocols were approved by the Henan University of Science and Technology of Biology Animal Use Ethics Committee. Collections will be carried out in accordance with local and international laws. No special permits are needed for the marine invertebrate species used in this work and no ethics approvals are required for this study because research on echinoderms is not subject to ethics regulation. The animals used in our experiments were maintained and treated in compliance with the guidelines specified by the Belgian Ministry of Trade and Agriculture.

\section{References}


bioRxiv preprint doi: https://doi.org/10.1101/2021.02.12.430961; this version posted February 14, 2021. The copyright holder for this preprint

(which was not certified by peer review) is the author/funder. All rights reserved. No reuse allowed without permission.

Delroisse et al. 2020

293 1. Han Q, Keesing JK, Liu D. A review of sea cucumber aquaculture, ranching, and stock 1. Han Q, Keesing JK, Liu D. A review of sea cucumber aquaculture, ranchement in China. Reviews in Fisheries Science \& Aquaculture. 2016;24(4):326-41. 2. Toral-Granda V, Lovatelli A, Vasconcellos M. Sea cucumbers: a global review of fisheries and trade: Food and Agriculture Organization of the United Nations Rome; 2008.

3. Yang L, Zheng L, Rao Y, Rao D, Rao D. Research progress of active components, health function and development of sea cucumber viscera. Journal of Food Safety and Quality. 2018;9(10):2426-32.

4. Van Dyck S, Gerbaux P, Flammang P. Elucidation of molecular diversity and body distribution of saponins in the sea cucumber Holothuria forskali (Echinodermata) by mass spectrometry. Comparative Biochemistry and Physiology Part B: Biochemistry and Molecular Biology. 2009;152(2):124-34.

5. Roggatz CC, González-Wangüemert M, Pereira H, Rodrigues MJ, da Silva MM, Barreira $L$, et al. First report of the nutritional profile and antioxidant potential of Holothuria arguinensis, a new resource for aquaculture in Europe. Natural product research. 2016;30(18):2034-40.

6. Bordbar S, Anwar F, Saari N. High-value components and bioactives from sea cucumbers for functional foods - a review. Marine drugs. 2011;9(10):1761-805.

7. Tian $F$, Zhang $X$, Tong $Y, Y i$, Zhang $S$, Li L, et al. PE, a new sulfated saponin from sea cucumber, exhibits anti-angiogenic and anti-tumor activities in vitro and in vivo. Cancer biology $\&$ therapy. $2005 ; 4(8): 874-82$.

8. Tuwo A, Conand C. Reproductive biology of the holothurian Holothuria forskali (Echinodermata). Journal of the Marine Biological Association of the United Kingdom. 1992;72(4):74558.

9. Zhou Z, Dong Y, Sun H, Yang A, Chen Z, Gao S, et al. Transcriptome sequencing of sea cucumber (Apostichopus japonicus) and the identification of gene-associated markers. Molecular ecology resources. 2014;14(1):127-38.

10. O'Hara TD, Hugall AF, Thuy B, Moussalli A. Phylogenomic resolution of the class Ophiuroidea unlocks a global microfossil record. Current Biology. 2014;24(16):1874-9.

11. Delroisse J, Ortega-Martinez O, Dupont S, Mallefet J, Flammang P. De novo transcriptome of the European brittle star Amphiura filiformis pluteus larvae. Marine genomics. 2015;23:109-21.

12. Purushothaman S, Saxena S, Meghah V, Swamy CVB, Ortega-Martinez O, Dupont S, et al. Transcriptomic and proteomic analyses of Amphiura filiformis arm tissue-undergoing regeneration. Journal of proteomics. 2015;112:113-24.

13. Delroisse J, Mallefet J, Flammang P. De novo adult transcriptomes of two European brittle stars: spotlight on opsin-based photoreception. PLoS One. 2016;11(4):e0152988.

14. Hennebert E, Wattiez R, Demeuldre M, Ladurner P, Hwang DS, Waite JH, et al. Sea star tenacity mediated by a protein that fragments, then aggregates. Proceedings of the National Academy of Sciences. 2014:201400089.

15. Zandawala M, Moghul I, Yañez Guerra LA, Delroisse J, Abylkassimova N, Hugall AF, et al. Discovery of novel representatives of bilaterian neuropeptide families and reconstruction of neuropeptide precursor evolution in ophiuroid echinoderms. Open biology. 2017;7(9):170129.

16. Grabherr MG, Haas BJ, Yassour M, Levin JZ, Thompson DA, Amit I, et al. Full-length transcriptome assembly from RNA-Seq data without a reference genome. Nature biotechnology. 2011;29(7):644.

17. Pertea G, Huang X, Liang F, Antonescu V, Sultana R, Karamycheva S, et al. TIGR Gene Indices clustering tools (TGICL): a software system for fast clustering of large EST datasets. Bioinformatics. 2003;19(5):651-2.

18. Gu S, Fang L, Xu X. Using SOAPaligner for short reads alignment. Current protocols in bioinformatics. 2013;44(1):11.. 1-.. 7.

19. Waterhouse RM, Seppey M, Simão FA, Manni M, loannidis P, Klioutchnikov G, et al. BUSCO applications from quality assessments to gene prediction and phylogenomics. Molecular biology and evolution. 2018;35(3):543-8.

20. Iseli $\mathrm{C}$, Jongeneel CV, Bucher $\mathrm{P}$, editors. ESTScan: a program for detecting, evaluating, and reconstructing potential coding regions in EST sequences. ISMB; 1999.

21. Claudel-Renard C, Chevalet C, Faraut T, Kahn D. Enzyme-specific profiles for genome annotation: PRIAM. Nucleic acids research. 2003;31(22):6633-9.

22. Chen T-W, Gan R-C, Fang Y-K, Chien K-Y, Liao W-C, Chen C-C, et al. FunctionAnnotator, a versatile and efficient web tool for non-model organism annotation. Scientific reports. 2017;7(1):1-9. 
bioRxiv preprint doi: https://doi.org/10.1101/2021.02.12.430961; this version posted February 14, 2021. The copyright holder for this preprint (which was not certified by peer review) is the author/funder. All rights reserved. No reuse allowed without permission.

Delroisse et al. 2020

350 23. Wessel GM, Berg L, Adelson DL, Cannon G, McClay DR. A molecular analysis of hyalin-a 351 substrate for cell adhesion in the hyaline layer of the sea urchin embryo. Developmental biology. 352

353

354

355

356 1998;193(2):115-26.

24. Hennebert E, Leroy B, Wattiez R, Ladurner P. An integrated transcriptomic and proteomic analysis of sea star epidermal secretions identifies proteins involved in defense and adhesion. Journal of proteomics. 2015;128:83-91. 
A. Unigene annotation

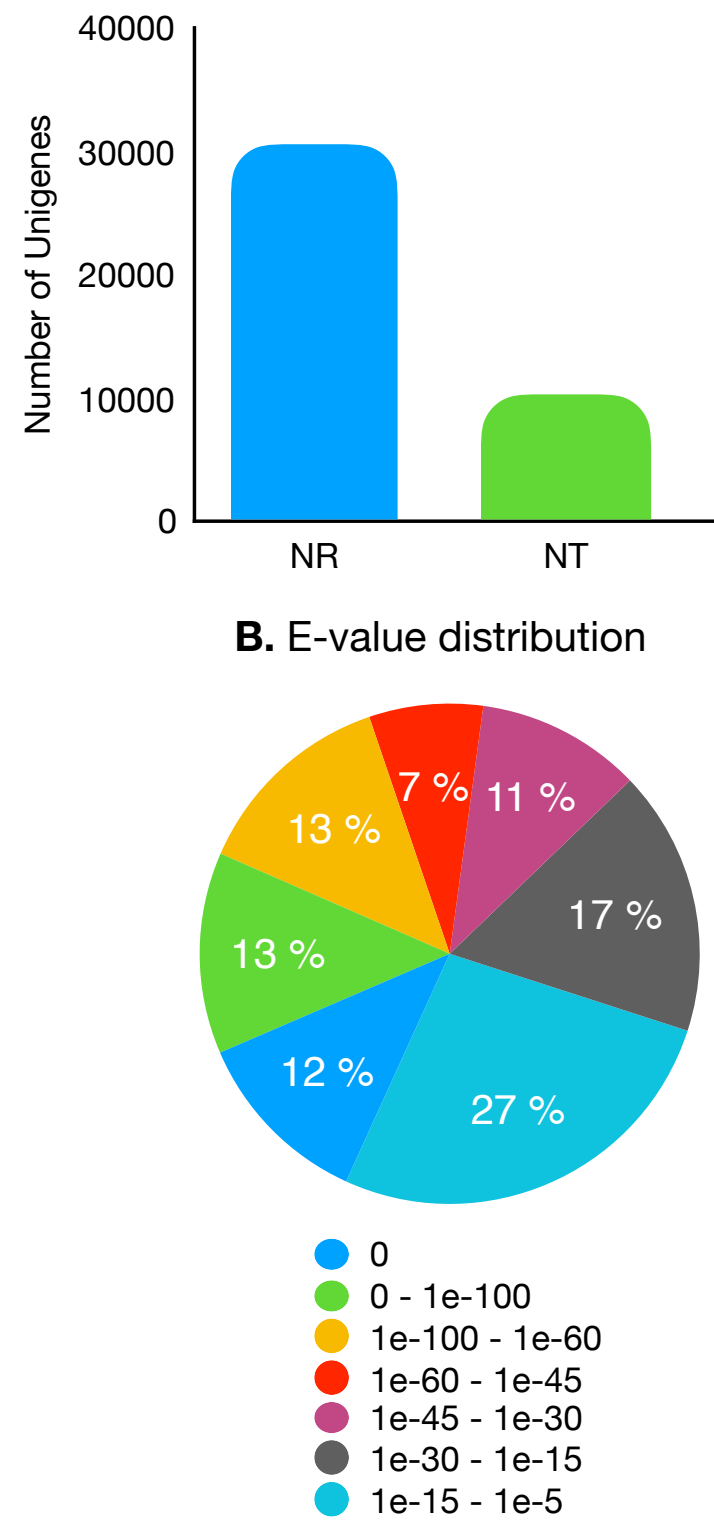

Swiss-Prot KEGG COG

GO

ALL

B. E-value distribution

C. Similarity distribution

D. Species distribution
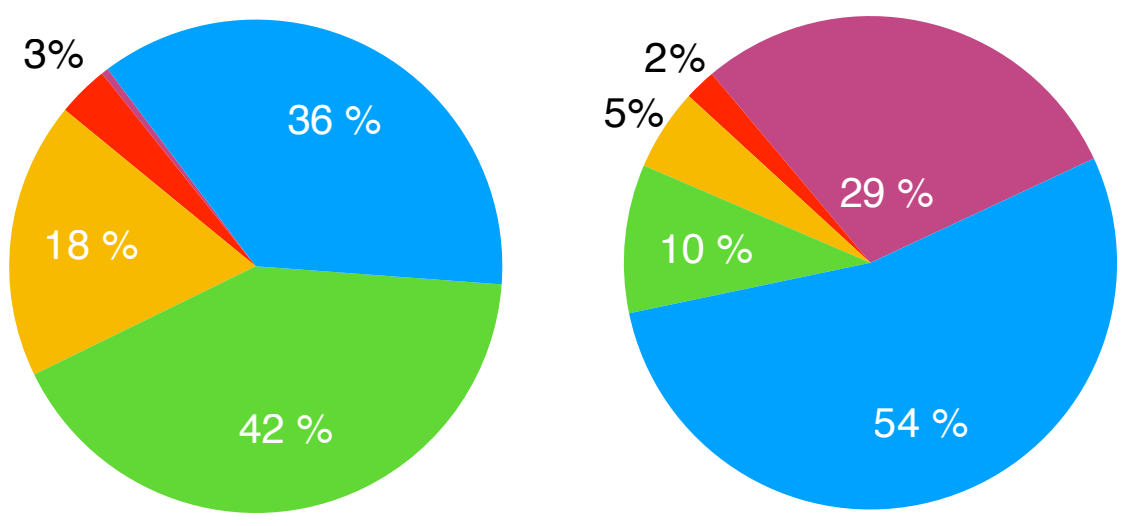

$16 \%-40 \%$

$40 \%-60 \%$

$60 \%-80 \%$

$80 \%-95 \%$

$95 \%-100 \%$
Strongylocentrotus purpuratus Saccoglossus kowalesvskii Amphioxus floridae Crassostrea gigas Others 


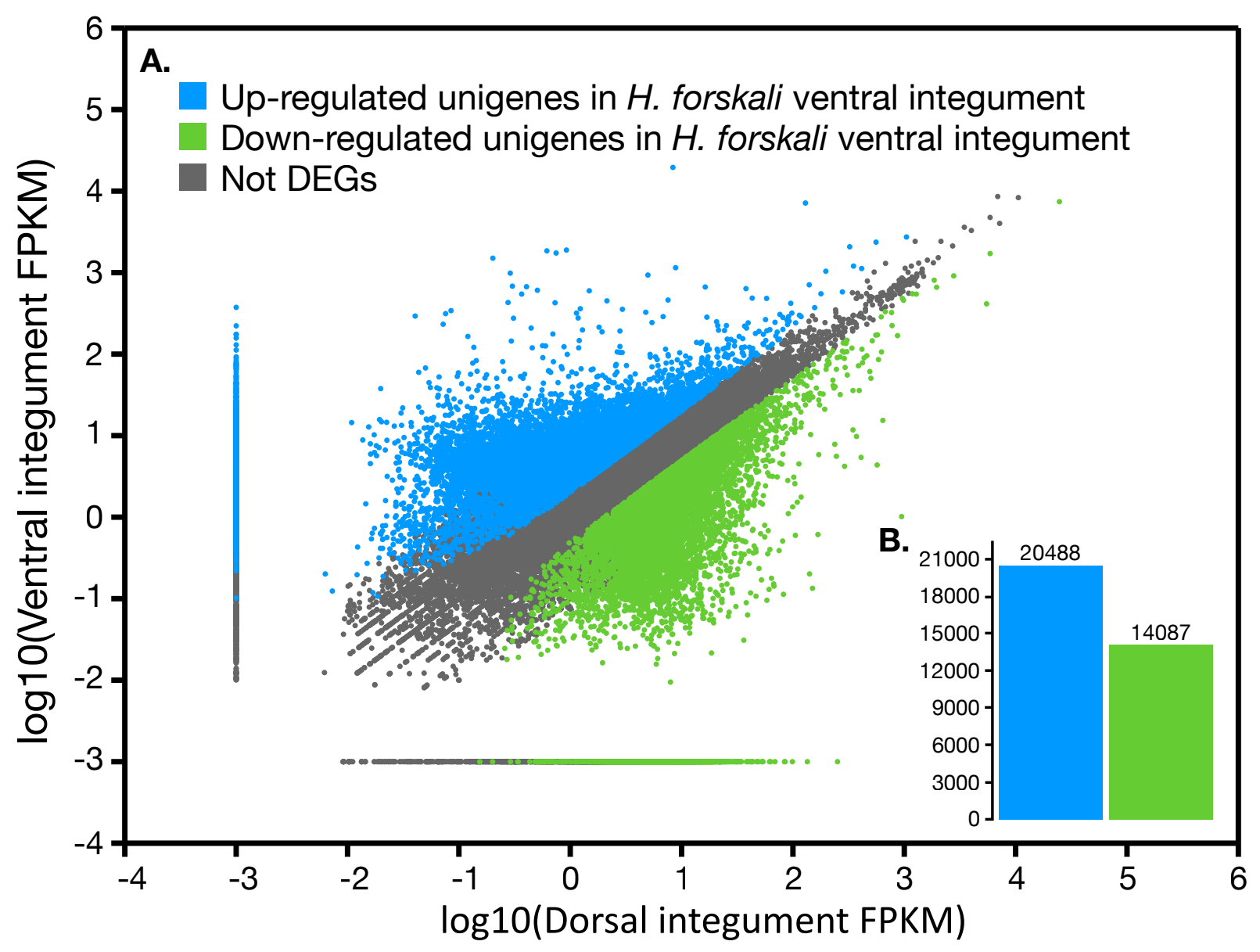

